\title{
Two Main Stripe Rust Resistance Genes Identified in Synthetic-Derived Wheat Line Soru\#1
}

\author{
Ruiqi Zhang, Ravi P. Singh, Morten Lillemo, Xinyao He, Mandeep S. Randhawa, \\ Julio Huerta-Espino, Pawan K. Singh, Zhikang Li, and Caixia Lan ${ }^{\dagger}$
}

First author: College of Agronomy/JCIC-MCP, Nanjing Agricultural University, Nanjing, P. R. China 210095; second, fourth, fifth, and seventh authors: International Maize and Wheat Improvement Center (CIMMYT), Apdo. Postal 6-641, México D.F., México 06600; third author: Department of Plant Sciences, Norwegian University of Life Sciences, P.O. Box 5003, As, Norway NO-1432; fourth author: Campo Experimental Valle de México INIFAP, Apdo. Postal 10, Chapingo, Edo. de México, México 56230; and eighth and ninth authors: College of Plant Science \& Technology, Huazhong Agricultural University, Wuhan, Hubei Province, P. R. China 430070.

Accepted for publication 26 July 2018.

\begin{abstract}
Stripe rust is a major disease constraint of wheat production worldwide. Resistance to stripe rust was analyzed using $131 \mathrm{~F}_{6}$ recombinant inbred lines (RILs) derived from a cross between synthetic derived wheat line Soru\#1 and wheat cultivar Naxos. The phenotype was evaluated in Mexico and Norway at both seedling and adult plant stages. Linkage groups were constructed based on 90K single-nucleotide polymorphism (SNP), sequence-tagged site, and simple sequence repeat markers. Two major resistance loci conferred by Soru\#1 were detected and located on chromosomes 1BL and 4DS. The 1BL quantitative trait loci explained 15.8 to 40.2 and $51.1 \%$ of the phenotypic variation at adult plant and seedling stages, respectively. This locus was
\end{abstract}

ABSTRACT identified as $Y r 24 / Y r 26$ based on the flanking markers and infection types. Locus 4DS was flanked by molecular markers D_GB5Y7FA02JMPQO_238 and BS00108770_51. It explained 8.4 to 27.8 and 5.5\% of stripe rust variation at the adult plant and seedling stages, respectively. The 4DS locus may correspond to known resistance gene $Y r 28$ based on the resistance source. All RILs that combine $Y r 24 / Y r 26$ and $Y r 28$ showed significantly reduced stripe rust severity in all four environments compared with the lines with only one of the genes. SNP marker BS00108770_51 was converted into a breederfriendly kompetitive allele-specific polymerase chain reaction marker that will be useful to accelerate $\operatorname{Yr} 28$ deployment in wheat breeding programs.
Wheat stripe or yellow rust, caused by Puccinia striiformis f. sp. tritici, is a major disease threatening wheat production worldwide. Stripe rust epidemics can rapidly destroy leaf tissues and significantly reduce grain yield and quality (Dubin and Brennan 2009). Although wheat stripe rust can be controlled by fungicides, planting resistant cultivars is considered a cost-effective and environmentally friendly approach to manage this disease. Stripe rust resistance genes can be classified into two categories based on the responses at different plant growth stages, such as seedling resistance and adult plant resistance (APR). Seedling resistance genes, often called allstage or major resistance genes, confer resistance at both seedling and adult plant stages, and are often race specific (Johnson 1981). APR genes are commonly detected at postseedling stages and can be further divided into two categories: race-specific APR genes that express a hypersensitive reaction at the adult plant stage (Gustafson and Shaner 1982) and race-nonspecific APR genes that are quantitatively inherited and show partial effects on disease severity with a compatible response against different pathotypes (Hautea et al. 1987).

Thus far, 82 stripe rust resistance genes have been cataloged in wheat (McIntosh et al. 2017) in combination with approximately 140 stripe rust resistance quantitative trait loci (QTL) summarized by Rosewarne et al. (2013). Among them, only three racenonspecific APR genes-that is, $\operatorname{Yr} 18$ (Spielmeyer et al. 2005),

†Corresponding author: C. Lan; E-mail: cxlan@mail.hzau.edu.cn

Funding: This work was supported by the Australian Grains Research and Development Corporation, the Australian Cereal Rust Control Program, and the National Natural Science Foundation of China (31301309). Field testing in Norway received funding from the Research Council of Norway (NFR project number 224833).

(C) 2019 The American Phytopathological Society
Yr29 (William et al. 2003), and Yr46 (Herrera-Foessel et al. 2011) have a pleiotropic effect on leaf rust, stem rust, and powdery mildew (Herrera-Foessel et al. 2014; Lillemo et al. 2008; Singh et al. 2013). Race-nonspecific APR genes usually cannot provide adequate resistance when they are present alone, whereas near-immunity resistance levels can be achieved by combining four to five minor APR genes in the same line (Singh et al. 2000a). In addition, pyramiding several APR genes with one to two seedling resistance genes to facilitate the development of effective resistance has been applied in wheat breeding for several years (Cox et al. 1994; Liu et al. 2000).

Synthetic hexaploid wheat is an artificial hybridization between tetraploid wheat (AABB) and accessions of Aegilops tauschii (DD). The International Maize and Wheat Improvement Center (CIMMYT) has developed a collection of synthetic wheat lines as a means of accessing traits in wheat wild relatives. Thus far, more than 1,000 synthetic wheat lines produced at CIMMYT have been widely distributed to breeding programs worldwide (Ortiz et al. 2008). Many A. tauschii accessions possess resistance to several diseases such as leaf rust, stem rust, and stripe rust and, in many cases, the resistances are functional when transferred into syntheticderived wheat (Liu et al. 2010; Ma et al. 1995; Singh et al. 2000b; Trethowan and van Ginkel 2009). Only a few stripe rust resistance genes have been mapped from the D genome in synthetic hexaploid wheat, such as $Y r 28$ (Singh et al. 2000b) and $Y r A S 2388$ (Huang et al. 2011) on 4DS and YrY206 (Zhang et al. 2008) and YrC108 (He et al. 2007) on chromosome 3D.

Synthetic-derived hexaploid wheat line Soru\#1 was developed at CIMMYT and showed good resistance to stripe rust and moderate resistance to Fusarium head blight (FHB) in Mexico and Norway (He et al. 2016). A major quantitative trait locus (QTL) for FHB resistance after spray and spawn inoculation was detected on chromosome arm 2DL in Soru\#1 (He et al. 2016); although Soru\#1 is susceptible to powdery mildew in the field, it harbors a minor 
QTL for powdery mildew resistance on 3AS (Windju et al. 2017). However, the genetic characterization of stripe rust resistance is unclear. The objectives of the present study were to characterize the stripe rust resistance genes in a Soru\# $1 \times$ Naxos population at both seedling and adult plant stages and to identify and develop molecular markers linked to resistance loci that can be utilized by breeding programs.

\section{MATERIALS AND METHODS}

Plant materials. A population of $131 \mathrm{~F}_{6}$ recombinant inbred lines (RILs) was developed from a cross between synthetic-derived hexaploid wheat line Soru\#1 with the German wheat cultivar Naxos (He et al. 2016). The pedigree of Soru\#1 is SABUF/5/BACANORA T 88/4/RABI//GANSO/CRANE/3/A. tauschii (190). Soru\#1 has good resistance to stripe rust, whereas Naxos is moderately susceptible in Mexico. The genotyping of this population was done using the Illumina iSelect 90K wheat chip (Wang et al. 2014) in Norway, as previously reported by $\mathrm{He}$ et al. (2016). After multiplication of the RILs and parents in Mexico, the seed were used for phenotyping stripe rust resistance in both field and greenhouse tests.

Greenhouse test. Seedling evaluations of Soru\#1, Naxos, and the RIL population were conducted in the greenhouse using the currently predominant Mexican $P$. striiformis f. sp. tritici isolate Mex14.191, which is virulent to resistance genes $\operatorname{Yr} 2,3,6,7,8,9$, (17), 27, 31, and 73+74 and avirulent to $\mathrm{Yrl}, 5,10,15$, and 24/26. In order to confirm the effect of $Y r 24 / Y r 26$, the RIL population was tested by using a new $P$. striiformis f. sp. tritici isolate, Mex16.04, that has avirulence and virulence similar to that of Mex14.191, except that it is also virulent to $\mathrm{Yr} 10$ and $\mathrm{Yr} 24 / \mathrm{Yr} 26$, based on the seedling responses of 30 differential lines with known stripe rust resistance genes (mostly in the Avocet background). Seedlings were inoculated at the two-leaf stage. Inoculated plants were placed in a dew chamber at $7^{\circ} \mathrm{C}$ for $24 \mathrm{~h}$ and then transferred to the greenhouse. Minimum, maximum, and mean postinoculation greenhouse temperatures were $10.8,22.6$, and $17.0^{\circ} \mathrm{C}$, respectively, for the Mex14.191 isolate and 9.1, 19.4, and $14.3^{\circ} \mathrm{C}$, respectively, for the Mex16.04 isolate. Infection type (IT) data were recorded approximately 14 days postinoculation based on a modified 0-to-9 scale according to the description by McNeal et al. (1971), where $0=$ no visible infection, $1=$ necrotic or chlorotic flecks without sporulation, 2 = necrotic or chlorotic stripes without sporulation, $3=$ necrotic or chlorotic stripes with trace sporulation, $4=$ necrotic or chlorotic stripes with light sporulation, $5=$ necrotic or chlorotic stripes with intermediate sporulation, 5-6 = necrotic or chlorotic stripes with intermediate to moderate sporulation, $6=$ chlorotic stripes with moderate sporulation, $7=$ stripes without chlorosis or necrosis and with moderate sporulation, $8=$ stripes without chlorosis or necrosis and with sufficient sporulation, $9=$ stripes without chlorosis or necrosis and abundant sporulation, 1-2 = small necrotic or chlorotic stripes without sporulation, and 6-7 = stripes with very little chlorosis and with sufficient sporulation. To analyze stripe rust seedling resistance genes, RILs with ITs 1 to 5 were considered resistant, those with types 7 to 8 were considered susceptible, and RILs with types 6 and 6-7 were considered intermediate and removed from final data analysis.

Field trials. Parents and RILs were evaluated for APR to stripe rust at CIMMYT's station in Toluca, Mexico, during the 2014, 2015, and 2016 growing seasons (hereinafter referred to as YR2014, YR2015, and YR2016M experiments, respectively). Field plots consisted of 70-cm paired rows with approximately 100 plants of each line. A mixture of wheat lines derived from an Avocet $\times$ Attila cross, Morocco, and an Avocet near-isogenic line for gene $\mathrm{Yr} 31$ used as a stripe rust spreader were sown around the experimental field and as hill plots in the middle of a $30-\mathrm{cm}$ pathway on one side of each RIL plot. A mixture of $P$. striiformis f. sp. tritici isolates Mex96.11 and Mex08.13 was sprayed during YR2014M onto the spreaders within and around the experimental areas. Isolate Mex08.13 is virulent on $\operatorname{Yr} 2,6,7,8,9,31$, and $73+74$, whereas Mex96.11 is virulent on $\operatorname{Yr} 2,3,6,7,9,27$, and 73+74 (Lan et al. 2014). P. striiformis f. sp. tritici isolate Mex14.191 was used in YR2015 and YR2016M. Disease severity was recorded when the flag leaves of the susceptible check Avocet displayed approximately $80 \%$ severity and then approximately 10 days later, based on the modified Cobb Scale (Peterson et al. 1948).

In the 2016 crop season, stripe rust phenotyping was also conducted at Vollebekk research station in As, Norway (referred to as YR2016N). The parents and the RILs were sown in hill plots following an $\alpha$-lattice design with two replicates. The nursery was naturally infected with stripe rust, and scoring was done based on

TABLE 2. Phenotypic Pearson's correlations for stripe rust in the Sor$\mathrm{u} \# 1 \times$ Naxos $\mathrm{F}_{6}$ recombinant inbred line populations using the final disease severity score for each environment ${ }^{\mathrm{a}}$

\begin{tabular}{lccc}
\hline Environment & Toluca 2014 & Toluca 2015 & Toluca 2016 \\
\hline Toluca 2015 & $0.81^{* *}$ & $\ldots$ & $\ldots$ \\
Toluca 2016 & $0.79^{* *}$ & $0.71^{* *}$ & $\ldots$ \\
As 2016 & $0.81^{* *}$ & $0.73^{* *}$ & $0.66^{* *}$ \\
\hline
\end{tabular}

a Asterisks (**) indicate $P<0.0001$.

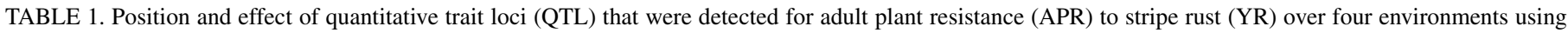

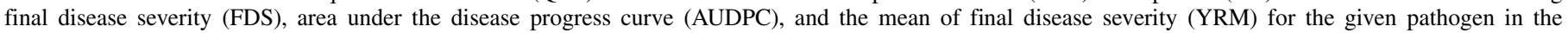
recombinant inbred line population ${ }^{\mathrm{a}}$

\begin{tabular}{|c|c|c|c|c|c|c|c|}
\hline Environment & Chr & Pos & Left marker & Right marker & LOD & PVE $(\%)$ & Add \\
\hline Toluca2014-FDS & $1 \mathrm{BL}$ & 39 & $T d \_c 31624 \_230$ & RAC875_c20775_540 & 12.2 & 33.6 & -8.6 \\
\hline Toluca2014-AUDPC & $1 \mathrm{BL}$ & 38 & $T d \_c 31624 \_230$ & RAC875_c20775_540 & 11.8 & 35.9 & -45.2 \\
\hline Toluca2015-FDS & $1 \mathrm{BL}$ & 39 & $T d \_c 31624 \_230$ & RAC875_c20775_540 & 6.9 & 18.9 & -9.2 \\
\hline Toluca2015-AUDPC & $1 \mathrm{BL}$ & 39 & $T d \_c 31624 \_230$ & RAC875_c20775_540 & 7.7 & 20.5 & -61.7 \\
\hline Toluca2016-FDS & $1 \mathrm{BL}$ & 38 & $T d \_c 31624 \_230$ & RAC875_c20775_540 & 7.2 & 15.8 & -11.7 \\
\hline Norway2016-FDS & $1 \mathrm{BL}$ & 39 & $T d \_c 31624 \_230$ & RAC875_c20775_540 & 14.2 & 40.2 & -1.9 \\
\hline Mean of FDS over four environments & $1 \mathrm{BL}$ & 39 & $T d \_c 31624 \_230$ & RAC875_c20775_540 & 13.5 & 25.5 & -8.9 \\
\hline Seedling Mex14.191 & $1 \mathrm{BL}$ & 39 & $T d \_c 31624 \_230$ & RAC875_c20775_540 & 26.8 & 51.1 & -2 \\
\hline Toluca2014-FDS & 4DS & 23 & D_GB5Y7FAO2JMPQO_238 & BS00108770_51 & 6 & 15 & -5.4 \\
\hline Toluca2014-AUDPC & 4DS & 23 & D_GB5Y7FA02JMPQO_238 & BS00108770_51 & 4.3 & 11.4 & -24 \\
\hline Toluca2015-FDS & 4DS & 23 & D_GB5Y7FA02JMPQ0_238 & BS00108770_51 & 9.2 & 26.3 & -10.1 \\
\hline Toluca2015-AUDPC & 4DS & 23 & D_GB5Y7FAO2JMPQO_238 & BS00108770_51 & 10.1 & 27.8 & -66.6 \\
\hline Toluca2016-FDS & 4DS & 23 & D_GB5Y7FA02JMPQO_238 & BS00108770_51 & 11.5 & 26.9 & -14.2 \\
\hline Norway2016-FDS & 4DS & 23 & D_GB5Y7FA02JMPQO_238 & BS00108770_51 & 3.6 & 8.4 & -0.8 \\
\hline Mean of FDS over four environments & 4DS & 23 & D_GB5Y7FA02JMPQO_238 & BS00108770_51 & 12 & 22.1 & -7.8 \\
\hline Seedling Mex14.191 & 4DS & 23 & D_GB5Y7FA02JMPQO_238 & BS00108770_51 & 4.4 & 5.5 & -0.6 \\
\hline Seedling Mex16.04 & 4DS & 23 & D_GB5Y7FA02JMPQ0_238 & BS00108770_51 & 21.1 & 54.7 & -1.4 \\
\hline
\end{tabular}

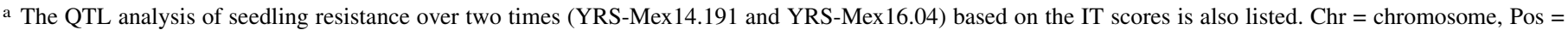
position, $\mathrm{LOD}=\log$ of the likelihood, and $\mathrm{PVE}=$ phenotypic variance explained. 

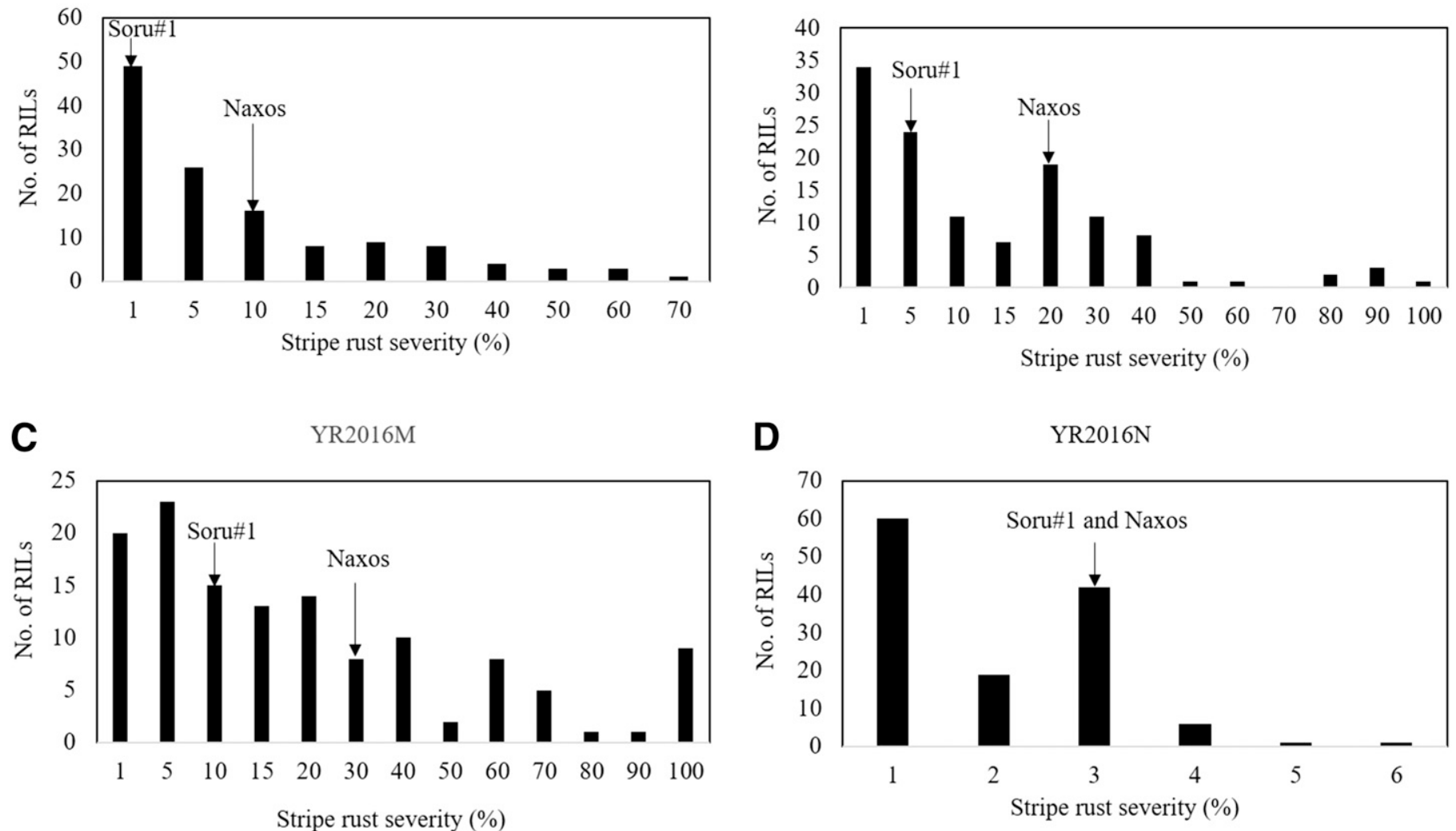

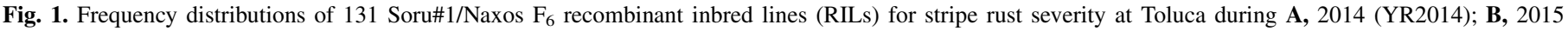
(YR2015); C, 2016 (YR2016M); and D, Norway in 2016 (YR2016N). Stripe rust severity for parents Soru\#1 and Naxos is indicated by arrows.

A

1BL

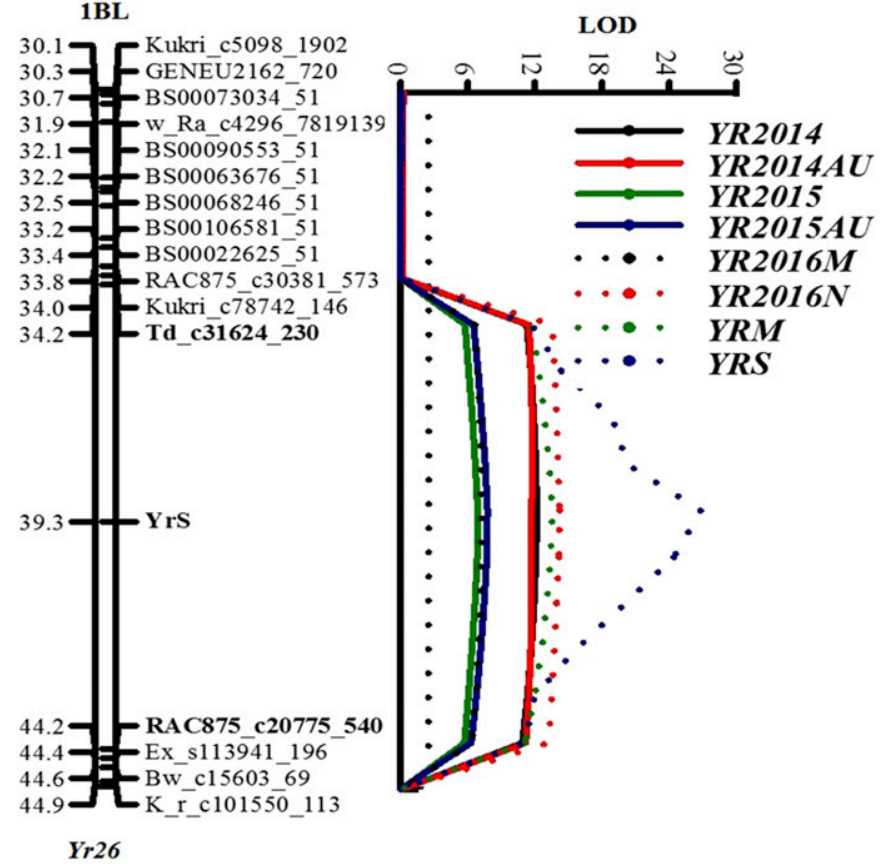

B

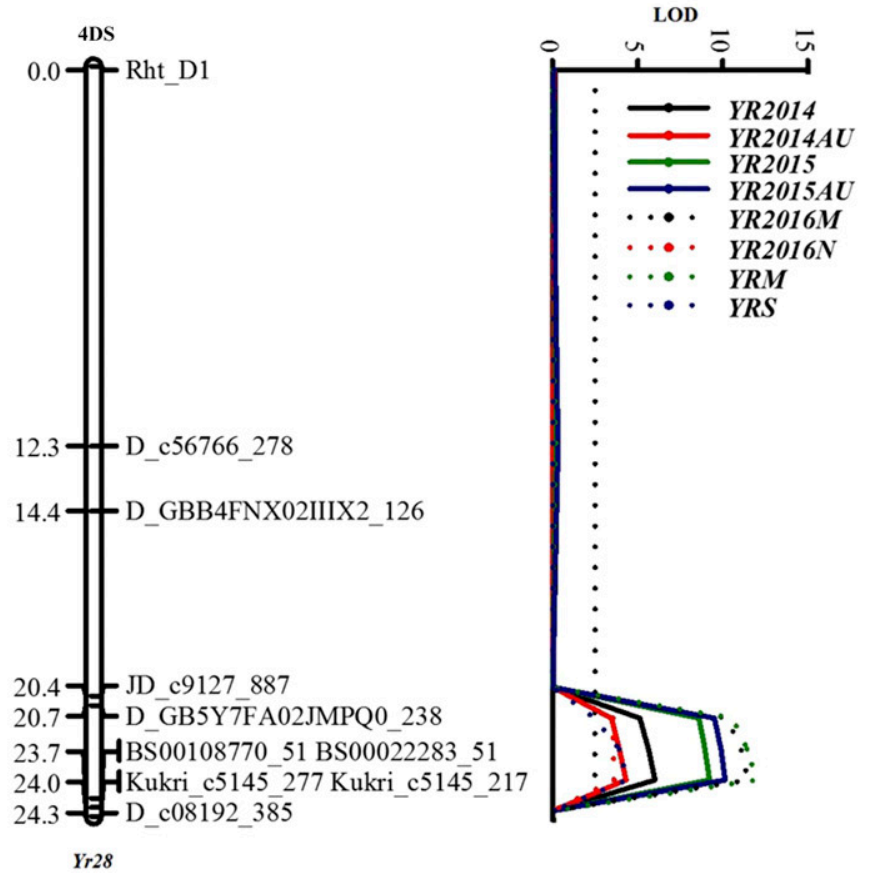

Fig. 2. Likelihood plots of quantitative trait loci (QTL) for adult plant and seeding resistance to stripe rust on chromosomes A, 1BL and B, 4DS, respectively, identified by IciMapping 4.1 in the Soru\#1/Naxos recombinant inbred line (RIL) population. Significant log of the likelihood (LOD) thresholds were detected based on 1,000 permutations. Positions (in centimorgans) of the molecular markers along chromosomes are shown on the vertical axis; accumulated genetic distances of the linkage groups are also shown. YR2014, YR2015, YR2016M, and YR2016N are the final disease severity (FDS) at Toluca in 2014, 2015, and 2016 in Norway, respectively. AU represents the area under the disease progress curve, YRM is the mean of FDS over test environments, and YRS is the infection type data at the seedling stage. QTL flanking markers are shown in bold. 
the percentage of infected flag leaf area approximately 2 weeks after heading. It was determined that the natural stripe rust epidemic consisted predominantly of the race lineages Warrior(-) and Triticale 2015 (Ali et al. 2017), based on the virulence analysis conducted by the Global Rust Reference Center at Aarhus University in Denmark of leaf samples collected from the field trial.

Genetic, statistical, and QTL analyses. The frequency distributions of 131 Soru\# $1 \times$ Naxos RILs for stripe rust severity and phenotypic Pearson's correlations were analyzed using IBM SPSS Statistics 22 software. Factorial analyses of variance (ANOVA) were conducted to test for the significance of interactions between detected resistance loci based on final disease severity (FDS).

Single-nucleotide polymorphism (SNP) marker data and linkage maps from previous studies by He et al. (2016) were utilized. For additional genotyping, the DNA of parents and RILs was extracted from approximately 20 plants per line using the cetyltrimethylammonium bromide method (Dreisigacker et al. 2016). To confirm mapped loci, sequence-tagged site marker $X W E 173$ specific to stripe rust resistance gene $Y r 24 / 26$ (Wang et al. 2008) and simplesequence repeat (SSR) marker Xwmc285 linked to $\mathrm{YrAS} 2388$ on chromosome 4DS (Huang et al. 2011) were used to genotype the parents and the whole population. In addition, flanking SNP markers were converted into Kompetitive allele specific polymerase chain reaction (KASP) markers and verified in the population using KASPar technology by LGC Genomics (https://www .lgcgroup.com/products/kasp-genotyping-chemistry/). Finally, 3,269 polymorphic markers were used to construct the linkage maps with Joinmap 4.1. Linkage maps were graphically visualized with MapChart 2.3. We conducted QTL mapping of FDS and calculated the area under the disease progress curve from each experiment, as well as the mean of final stripe rust severity across experiments, to detect the positions of QTL using inclusive composite interval mapping (ICIM) (Meng et al. 2015). Log of the likelihood (LOD) scores were calculated from 1,000 permutations for each trait to determine significant QTL. The percentages of phenotypic variance

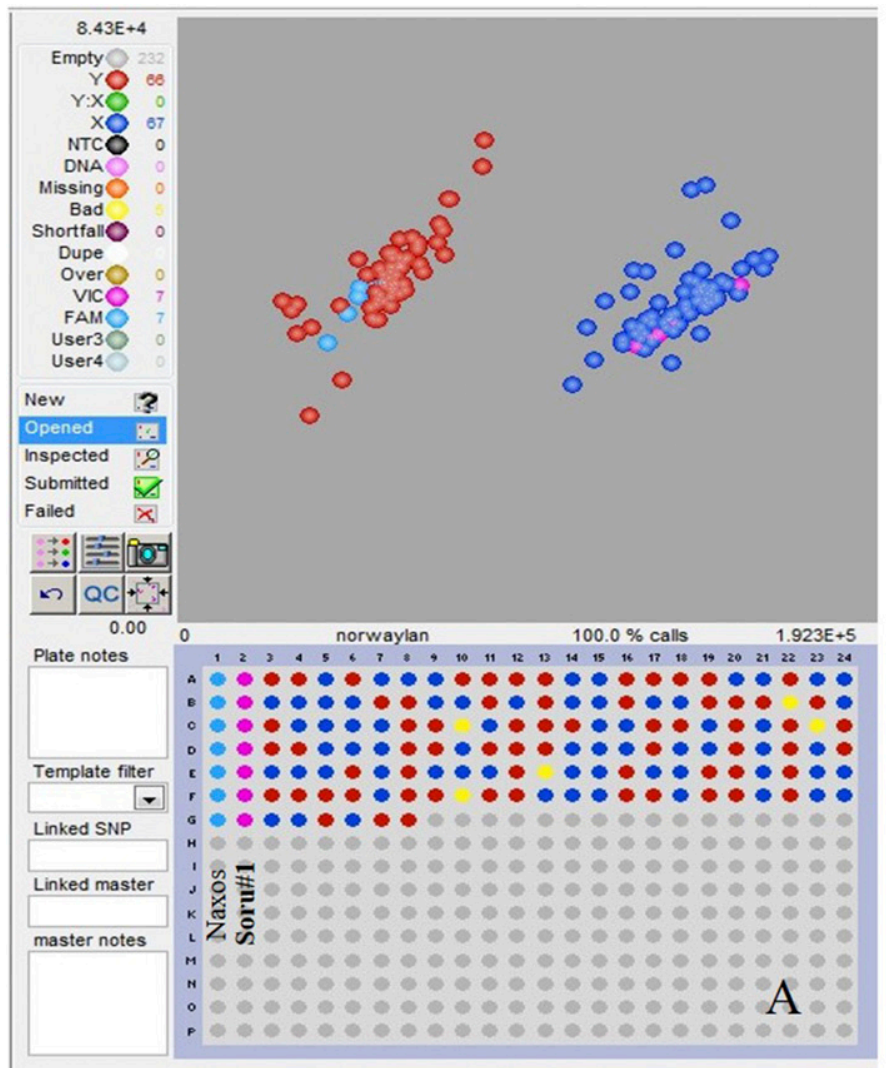

explained by individual QTL and the additive effects at the LOD peaks were obtained though stepwise regression with IciMapping 4.1.

\section{RESULTS}

Seedling resistance analysis. The ITs against Mexican P. striiformis f. sp. tritici isolate Mex14.191 were 1-2 and 6-7 for the synthetic wheat derivative Soru\#1 and Naxos, respectively. ITs of progenies ranged from 1 to 8 . RILs assigned based on IT scores showed a distribution of 77:34 resistant/susceptible lines, indicating distorted segregation for stripe rust resistance in the population $(P<$ 0.0001). ICIM identified two QTL derived from Soru\#1 based on seedling ITs. A major QTL located on chromosome 1BL was flanked by markers Td_c31624_230 and RAC875_c20775_540 and explained $51.1 \%$ of the phenotypic variance (Table 1$)$. It was likely Yr24/Yr26 based on the closely linked molecular marker XWE173. The ITs of RILs with the Yr24/Yr26-positive allele ranged from 1 to 3 , whereas they ranged from 6 to 8 for RILs with $Y r 24 / Y r 26$ and $Y r 28$ (the second gene segregating in the population) negative alleles. Another minor QTL was mapped on chromosome arm 4DS and located in the interval between markers D_GB5Y7FA02JMPQ0_238 and BS00108770_51. It explained 5.5\% of stripe rust IT variation (Table 1). It was likely $\mathrm{Yr} 28$ based on the chromosome location and

TABLE 3. Sequence of kompetitive allele-specific polymerase chain reaction (KASP) marker BS00108770_51

\begin{tabular}{ll}
\hline KASP & \multicolumn{1}{c}{ BS00108770_51 } \\
\hline Primer_AlleleFAM & CTCCTGCCCCTTGGCGGT \\
Primer_AlleleHEX & CTCCTGCCCCTTGGCGGC \\
Primer_Common & CTGAGGGGGAGAGTTTGCCCTA \\
AlleleFAM & A \\
AlleleHEX & $\mathrm{G}$ \\
\hline
\end{tabular}

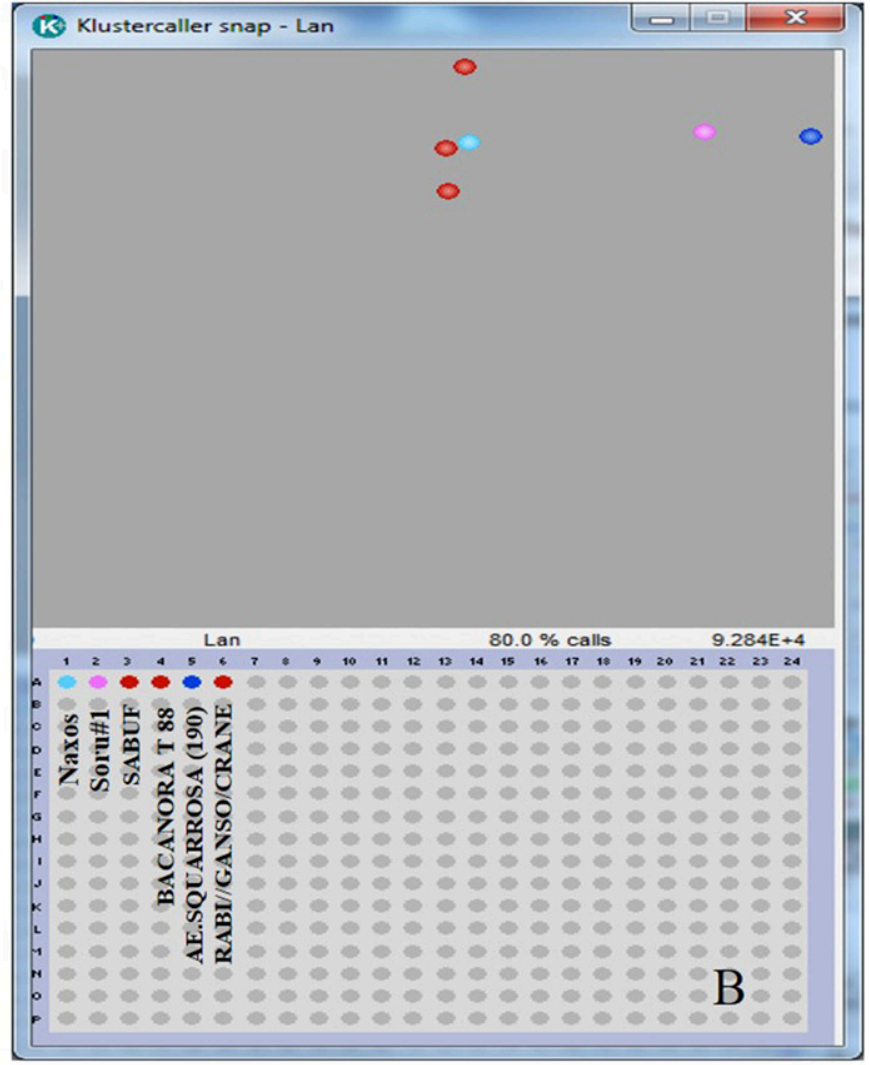

Fig. 3. Clustering pattern of Kompetitive allele-specific polymerase chain reaction marker BS00108770_51 genotyped on A, the population and B, parents. 
resistance source. The ITs of RILs with the $\operatorname{Yr} 28$ positive allele ranged from 3 to 6 , whereas they ranged from 6 to 8 for RILs with the $\operatorname{Yr} 28$ negative allele against Mex14.191. In addition, the RIL population was tested with $Y r 24 / Y r 26$ virulent $P$. striiformis f. sp. tritici isolate Mex16.04 at the seedling stage. QTL analysis only mapped one seedling resistance locus on chromosome 4DS which was flanked by the same molecular markers as $\mathrm{Yr} 28$ mentioned above, whereas the 1BL QTL was not detected against the Mex16.04 isolate as we expected (Table 1). The ITs of RILs with the $\mathrm{Yr} 28$ positive allele ranged from 1 to 4 , whereas they ranged from 6 to 8 for RILs with the $Y r 28$ negative allele against Mex16.04. With this $Y r 24 /$ Yr26 virulent isolate, the $Y r 28$ QTL explained $54.7 \%$ of stripe rust IT variation (Table 1).

Phenotypic analysis at the adult plant stage. Correlation coefficients $(r)$ of stripe rust severities in the Soru\# $1 \times$ Naxos population ranged from 0.66 to 0.81 and were significant among different environments and years (Table 2), indicating that the FDS of RILs were quite consistent over the four environments. The stripe rust severities for Soru\#1 and Naxos over four environments were 1 to 10 and 10 to $30 \%$, respectively, whereas the severities for RILs ranged from 1 to $90 \%$ (Fig. 1). The frequency distribution of RILs for stripe rust severity was continuous with a pronounced skew toward resistance (Fig. 1), suggesting that large-effect stripe rust resistance genes might be present in the RIL population.

QTL analysis at the adult plant stage. ICIM analysis mapped two major stripe rust resistance QTL in the present population and both of them were derived from Soru\#1 (Table 1; Fig. 2). The first QTL was located on chromosome 1BL and flanked by SNP markers $T d \_c 31624 \_230$ and RAC875_c20775_540. This QTL explained
15.8 to $40.2 \%$ of the severity variance across four environments (Table 1). The other major QTL located on chromosome arm 4DS was flanked by markers D_GB5Y7FA02JMPQ0_238 and BS00108770_51 (Table 1; Fig. 2B). It explained 8.4 to $27.8 \%$ of disease severity variance across the test environments. SNP marker BS00108770_51 was converted into a breeder-friendly KASP marker (Fig. 3A; Table 3), and we obtained the same genotyping results as with the 90K SNP marker. The genotype of KASP marker BS00108770_51 for Soru\#1's parents showed that A. tauschii (190) had the same allele as Soru\#1, whereas the other parents had the Naxos allele (Fig. 3B).

To estimate the effects of the QTL as well as their interactions on stripe rust severity at the adult plant stage, a two-way FACTOR ANOVA was carried out to test the principal effects of the single genes and their interactions on the FDS values. The flanking molecular markers for two resistance loci were used to infer the presence of parental alleles in each RIL. The 1BL QTL explained $13.2 \%$ of the variation, whereas 4DS QTL explained $16.5 \%$ of the variation. The interaction between the two loci was also significant and explained $10 \%$ of the variation (Table 4 ), indicating a synergistic effect on stripe rust resistance (Fig. 4).

\section{DISCUSSION}

The synthetic-derived line Soru\#1 was highly resistant to stripe rust in both the Mexican and Norwegian environments. Our results indicated that APR to stripe rust in Soru\#1 was controlled by two resistance loci located on chromosome arms 1BL and 4DS. The $1 \mathrm{BL}$ QTL is most likely the known stripe rust seedling resistance

TABLE 4. Two-way FACTOR analyses of variance of final disease severity (FDS) for two resistance genes, using environments as blocks

\begin{tabular}{|c|c|c|c|c|c|c|c|}
\hline Source & Number of RILs ${ }^{a}$ & DF & Type III SS & Mean square & $F$ value & $\operatorname{Pr}>F$ & Variation (\%) \\
\hline Environments & & 3 & 40675.3 & 13558.43 & 56.86 & $<0.0001$ & 0.183 \\
\hline Yr24 & 35 & 2 & 14626.33 & 7313.165 & 30.67 & $<0.0001$ & 0.132 \\
\hline Yr28 & 18 & 2 & 15114.31 & 7557.156 & 31.69 & $<0.0001$ & 0.165 \\
\hline
\end{tabular}

${ }^{\mathrm{a}}$ RILs = recombinant inbred lines.

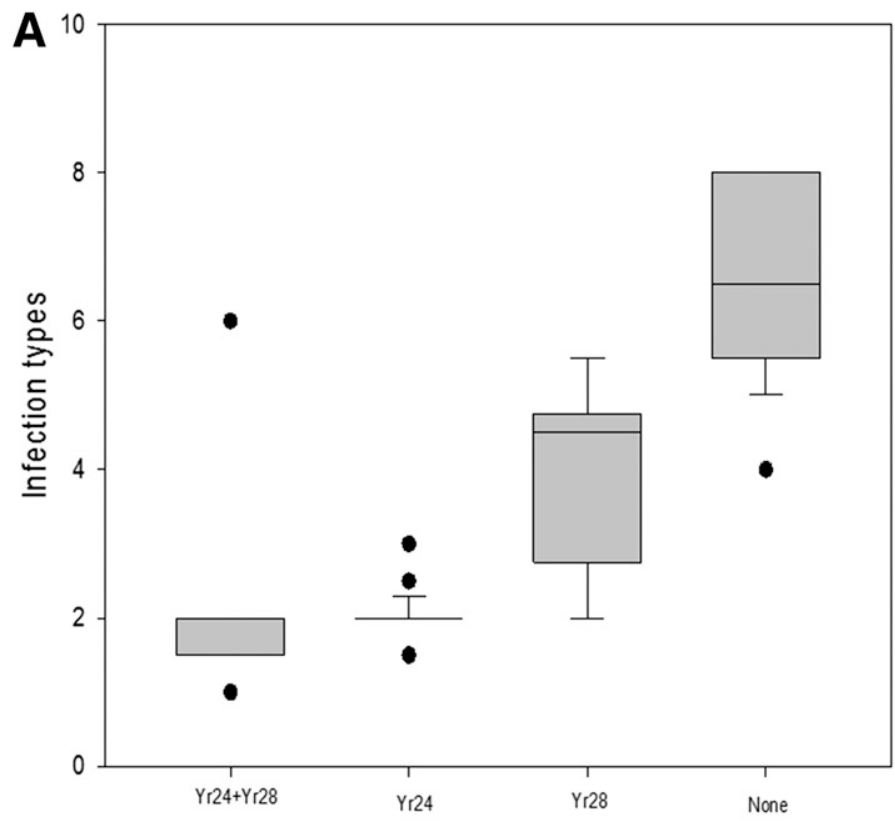

Gene combinations for Mex14.191

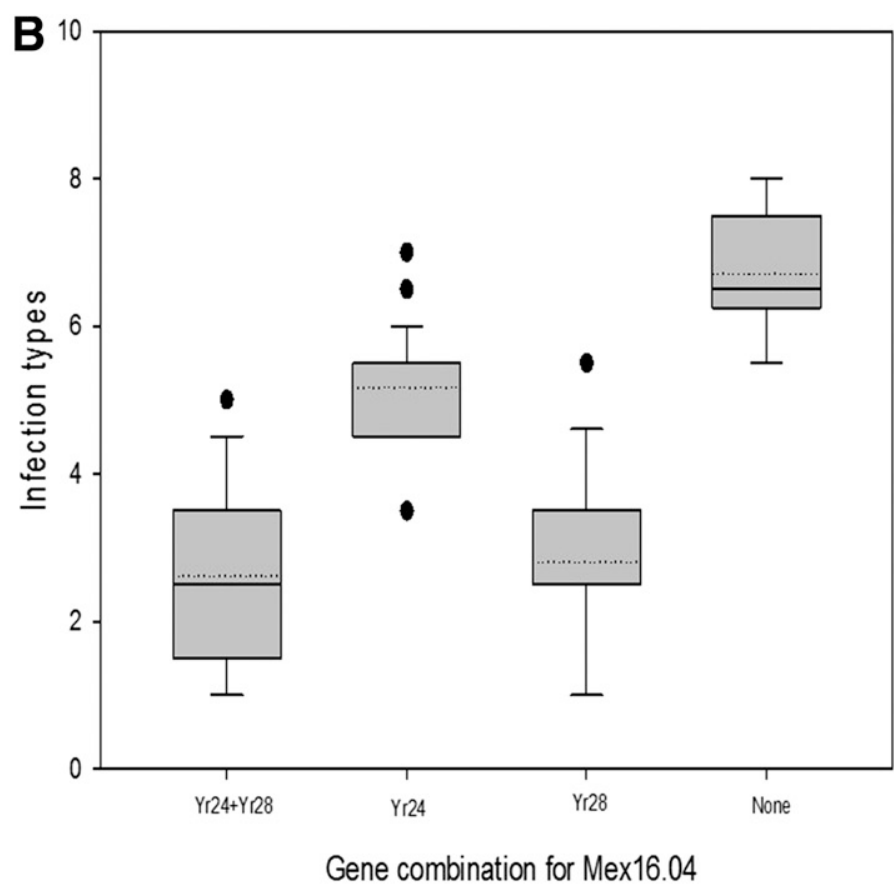

Fig. 4. Boxplot of gene interaction between $Y r 24$ and Yr28 with two Puccinia striiformis f. sp. tritici isolates A, Mex14.191 and B, Mex16.04, respectively, in the Soru\#1/Naxos population. 
gene $Y r 24 / Y r 26$ based on the seedling IT against both avirulent P. striiformis f. sp. tritici isolate Mex14.191 and virulent isolate Mex16.04 as well as the new $\operatorname{Yr} 24 / Y r 26$ closely linked molecular marker, WRS414, published by Wu et al. (2018). The strong effect of this QTL in the Norwegian environment (Table 1) is also consistent with the fact that the predominant race in that environment, Warrior(-), is avirulent to $Y r 24 / Y r 26$ (Ali et al. 2017). The $Y r 24$ gene originating from $T$. turgidum $\mathrm{L}$., has been widely deployed in wheat breeding programs by utilizing synthetic wheats as parents. Approximately 30 commercial varieties carrying this gene have been released and grown on over 3.4 million ha in China (Zhang et al. 2013). Unfortunately, $\operatorname{Yr} 24 / Y r 26$ has not provided adequate protection in recent years in China due to the presence of a new virulent stripe rust race, V26 (Liu et al. 2010). In 2016, virulence to $\mathrm{Yr} 24 / \mathrm{Yr} 26$ was detected in Mexico as well. Therefore, this resistance gene can no longer be used alone as a stripe rust resistance source in breeding programs.

Thus far, only one moderately effective seedling stripe rust resistance gene, $Y r 28$, derived from A. tauschii accession W-219, has been mapped on chromosome arm 4DS using a RIL population derived from a cross of synthetic hexaploid wheat and T. aestivum 'Opata 85' (Singh et al. 2000b). However, no polymerase chain reaction (PCR) marker is available to identify $\mathrm{Yr} 28$. Thus, the relationship between the resistance genes on 4DS and $Y r 28$ needs to be confirmed using an allelism test even though the phenotypic expression of resistance was similar. Genetic analysis for this purpose is underway. Huang et al. (2011) also mapped a stripe rust resistance gene (YrAS2388) on the terminal part of chromosome arm 4DS within 1.7 centimorgan of SSR marker Xwmc285 in A. tauschii accession AS2388. The SSR marker Xwmc285 was genotyped in this study but no polymorphism was detected between Soru\#1 and Naxos, indicating that the resistance gene on 4DS in

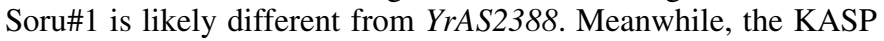
marker (BS00108770_51) linked to the 4DS resistance gene identified in our study can be used to deploy this locus in wheat breeding using marker-assisted selection.

Effective APR to wheat stripe rust can be achieved by pyramiding genes that, alone, may be only partially effective but, combined together, provide effective resistance to the current pathogen population (Castro et al. 2003). Gene pyramiding has been effective for prolonging stripe rust resistance in wheat. For example, a synergistic interaction between $\mathrm{Yr} 36$ and $\mathrm{Yr} 18$ on stripe rust severity was inferred from the observations of transferring $\mathrm{Yr} 36$ into different wheat backgrounds (Uauy et al. 2005). Molecular markers genetically linked to stripe rust resistance genes or QTL facilitate gene pyramiding and accurate incorporation of resistance into new germplasm. For the Soru\#1 $\times$ Naxos RIL population, we have shown the presence of two loci for stripe rust resistance which can be identified through molecular markers and incorporated into wheat varieties. The combination of resistance genes on $1 \mathrm{BL}$ (shown to be $Y r 24 / Y r 26$ ) and 4DS (likely $Y r 28$ ) revealed that the interaction of two different seedling genes might have contributed to the larger reduction in stripe rust severity of Soru\#1 to the avirulent races used in Mexico and Norway. This result supports the hypothesis that the pyramiding of seedling genes may also be a good strategy to maintain low levels of stripe rust severity (Zheng et al. 2017). Cultivars with Yr24/Yr26 have been rapidly released in China but many commercial varieties and elite lines containing this gene alone cannot give adequate stripe rust resistance because a new stripe rust race (V26) is virulent to this locus (Liu et al. 2010; Yang et al. 2016). The resistance gene on 4DS provides an additional gene that wheat breeders can pyramid with the already used $\mathrm{Yr} 24 / \mathrm{Yr} 26$ gene, especially in areas where $\mathrm{Yr} 24 / \mathrm{Yr} 26$ virulence is absent in the pathogen population. However, further studies are needed to determine whether pyramiding the two major QTL is appropriate to reduce stripe rust without affecting yield and other agronomic traits in different target environments.

\section{ACKNOWLEDGMENTS}

We thank Z. Kang, D. Han, and J. Wu from Northwest A\&F University, China, for providing the KASP marker wrs 414 and related working space to test Soru\#1/Naxos population; and A. McNab and M. Listman for technical editing.

\section{LITERATURE CITED}

Ali, S., Rodriguez-Algaba, J., Thach, T., Sorensen, C. K., Hansen, J. G., Lassen, P., Nazari, K., Hodson, D. P., Justesen, A. F., and Hovmoller, M. S. 2017. Yellow rust epidemics worldwide were caused by pathogen races from divergent genetic lineages. Front. Plant Sci. 8:1057.

Castro, A. J., Chen, X. M., Hayes, P. M., and Johnston, M. 2003. Pyramiding quantitative trait locus (QTL) alleles determining resistance to barley stripe rust: Effects on resistance at the seedling stage. Crop Sci. 43:651-659.

Cox, T. S., Raupp, W. J., and Gill, B. S. 1994. Leaf rust-resistance genes, Lr41, Lr42 and Lr43 transferred from Triticum tauschii to common wheat. Crop Sci. 34:339-343.

Dreisigacker, S., Sehgal, D., Reyes-Jaimez, A. E., Luna-Garrido, B., Muñoz-Zavala, S., Núñez-Ríos, C., Mollinsm, J., and Mall, S., eds. 2016. CIMMYT Wheat Molecular Genetics: Laboratory Protocols and Applications to Wheat Breeding. CIMMYT, D.F., México.

Dubin, H. J., and Brennan, J. P. 2009. Fighting a "Shifty Enemy": The international collaboration to contain wheat rusts. Pages 19-24 in: Millions Fed: Proven Successes in Agricultural Development. D. J. Spielman and R. Pandya-Lorch, eds. International Food Policy Research Institute, Washington, DC.

Gustafson, G., and Shaner, G. 1982. Influence of plant age on the expression of slow-mildewing resistance in wheat. Phytopathology 72:746-749.

Hautea, R., Coffman, W., Sorrells, M., and Bergstrom, G. 1987. Inheritance of partial resistance to powdery mildew in spring wheat. Theor. Appl. Genet. 73:609-615.

He, M. Z., Wang, L. M., Zhang, Z. Y., Xu, S. C., Wang, L. L., and Xin, Z. Y. 2007. Identification and molecular mapping of a novel stripe rust resistance gene in a Triticum durum-Aegilops tauschii amphiploid CI108. Acta Agron. Sin. 33:1045-1050.

He, X., Lillemo, M., Shi, J., Wu, J., Bjørnstad, Å., Belova, T., Dreisigacker, S., Duveiller, E., and Singh, P. 2016. QTL Characterization of Fusarium head blight resistance in CIMMYT bread wheat line Soru\#1. PLoS One 11: e0158052.

Herrera-Foessel, S. A., Lagudah, E. S., Huerta-Espino, J., Hayden, M. J., Bariana, H. S., Singh, D., and Singh, R. P. 2011. New slow rusting leaf rust and stripe rust resistance genes $\operatorname{Lr67}$ and $\mathrm{Yr} 46$ in wheat are pleiotropic or closely linked. Theor. Appl. Genet. 122:239-249.

Herrera-Foessel, S. A., Singh, R. P., Lillemo, M., Huerta-Espino, J., Bhavani, S., Singh, S., Lan, C. X., Calvo-Salazar, V., and Lagudah, E. S. 2014. Lr67/ Yr46 confers adult plant resistance to stem rust and powdery mildew in wheat. Theor. Appl. Genet. 127:781-789.

Huang, L., Zhang, L. Q., Liu, B. L., Yan, Z. H., Zhang, B., Zhang, H. G., Zheng, Y. L., and Liu, D. C. 2011. Molecular tagging of a stripe rust resistance gene in Aegilops tauschii. Euphytica 179:313-318.

Johnson, R. 1981. Durable resistance: Definition, genetic control, and attainment in plant breeding. Phytopathology 71:567-568.

Lan, C. X., Singh, R. P., Huerta-Espino, J., Calvo-Salazar, V., and Herrera-Foessel, S. A. 2014. Genetic analysis of resistance to leaf rust and stripe rust in wheat cultivar Francolin\#1. Plant Dis. 98:1227-1234.

Lillemo, M., Asalf, B., Singh, R. P., Huerta-Espino, J., Chen, X. M., He, Z. H., and Bjørnstad, A. 2008. The adult plant rust resistance loci Lr34/Yrl8 and Lr46/Yr29 are important determinants of partial resistance to powdery mildew in bread wheat line Saar. Theor. Appl. Genet. 116:1155-1166.

Liu, D. C., Zhang, L. Q., Yan, Z. H., Lan, X. J., and Zheng, Y. L. 2010. Stripe rust resistance in Aegilops tauschii and its genetic analysis. Genet. Resour. Crop Evol. 57:325-328.

Liu, J., Liu, D., Tao, W., Li, W., Wang, S., Chen, P., Cheng, S., and Gao, D. 2000. Molecular marker-facilitated pyramiding of different genes for powdery mildew resistance in wheat. Plant Breed. 119:21-24.

Liu, T. G., Peng, Y. L., Chen, W. Q., and Zhang, Z. Y. 2010. First detection of virulence in Puccinia striiformis f. sp. tritici in China to resistance genes Yr24 (= Yr26) present in wheat cultivar Chuanmai 42. Plant Dis. 94:1163.

Ma, H., Singh, R. P., and Mujeeb-Kazi, A. 1995. Resistance to stripe rust in Triticum turgidum, T. tauschii and their synthetic hexaploids. Euphytica 82: 117-124.

McIntosh, R. A., Dubcovsky, J., Rogers, W. J., Morris, C., and Xia, X. C. 2017. Catalogue of Gene Symbols for Wheat: 2017 Supplement. https:// shigen.nig.ac.jp/wheat/komugi/genes/macgene/supplement2017.pdf

McNeal, F. H., Koebner, C. F., Smith, E. P., Tate, W. S., and Russell, T. S. 1971. A Uniform System for Recording and Processing Cereal Research 
Data. United States Department of Agriculture-Agricultural Research Service Bull. 34-121.

Meng, L., Li, H., Zhang, L., and Wang, J. 2015. QTL IciMapping: Integrated software for genetic linkage map construction and quantitative trait locus mapping in biparental populations. Crop J. 3:269-283.

Ortiz, R., Braun, H., Crossa, J., Crouch, J., Davenport, G., Dixon, J., Dreisigacker, S., Duveiller, E., He, Z. H., Huerta, J., Joshi, A., Kishii, M., Kosina, P., Manes, Y., Mezzalama, M., Morgounov, A., Murakami, J., Nicol, J., Ferrara, G., Ortiz-Monasterio, J., Payne, T., Pena, R., Reynolds, M., Sayre, K., Sharma, R., Singh, R., Wang, J., Warburton, M., Wu, H., and Iwanaga, M. 2008. Wheat genetic resources enhancement by the International Maize and Wheat Improvement Center (CIMMYT). Genet. Resour. Crop Evol. 55:1095-1140.

Peterson, R. F., Campbell, A. B., and Hannah, A. E. 1948. A diagrammatic scale for estimating rust intensity on leaves and stems of cereals. Can. J. Res. 26c:496-500.

Rosewarne, G. M., Herrera-Foessel, S. A., Singh, R. P., Huerta-Espino, J., Lan, C. X., and He, Z. H. 2013. Quantitative trait loci of stripe rust resistance in wheat. Theor. Appl. Genet. 126:2427-2449.

Singh, R. P., Herrera-Foessel, S. A., Huerta-Espino, J., Lan, C. X., Basnet, B. R., Bhavani, S., and Lagudah, E. S. 2013. Pleiotropic gene Lr46/Yr29/ Pm39/Ltn2 confers slow rusting, adult plant resistance to wheat stem rust fungus. Pages 19-22 in: Proc. Borlaug Global Rust Initiative, 2013 Tech. Workshop, New Delhi, India.

Singh, R. P., Huerta-Espino, J., and Rajaram, S. 2000a. Achieving nearimmunity to leaf and stripe rusts in wheat by combining slow rusting resistance genes. Acta Phytopathol. Entomol. Hung. 35:133-139.

Singh, R. P., Nelson, J. C., and Sorrells, M. E. 2000b. Mapping Yr28 and other genes for resistance to stripe rust in wheat. Crop Sci. 40:1148-1155.

Spielmeyer, W., McIntosh, R. A., Kolmer, J., and Lagudah, E. S. 2005. Powdery mildew resistance and $\operatorname{Lr} 34 / \mathrm{Yr} 18$ genes for durable resistance to leaf and stripe rust cosegregate at a locus on the short arm of chromosome 7D of wheat. Theor. Appl. Genet. 111:731-735.

Trethowan, R., and van Ginkel, M. 2009. Synthetic wheat- an emerging genetic resource. Pages 369-385 in: Wheat Science and Trade. B. Carver, ed. Wiley-Blackwell, Ames, IA.

Uauy, C., Brevis, J. C., Chen, X. M., Khan, I., Jackson, L., Chicaiza, O., Distelfeld, A., Fahima, T., and Dubcovsky, J. 2005. High-temperature adultplant (HTAP) stripe rust resistance gene Yr36 from Triticum turgidum ssp. dicoccoides is closely linked to the grain protein content locus Gpc-B1. Theor. Appl. Genet. 112:97-105.
Wang, C. M., Zhang, Y. P., Han, D. J., Kang, Z. S., and Chen, P. D. 2008. SSR and STS markers for wheat stripe rust resistance gene $\operatorname{Yr} 26$. Euphytica 159: 359-366.

Wang, S., Wong, D., Forrest, K., Allen, A., Chao, S., Huang, B. E., Maccaferri, M., Salvi, S., Milner, S. G., Cattivelli, L., Mastrangelo, A. M., Whan, A., Stephen, S., Barker, G., Wieseke, R., Plieske, J., International Wheat Genome Sequencing Consortium, Lillemo, M., Mather, D., Appels, R., Dolferus, R., Brown-Guedira, G., Korol, A., Akhunova, A. R., Feuillet, C., Salse, J., Morgante, M., Pozniak, C., Luo, M. C., Dvorak, J., Morell, M., Dubcovsky, J., Ganal, M., Tuberosa, R., Lawley, C., Mikoulitch, I., Cavanagh, C., Edwards, K. J., Hayden, M., and Akhunov, E. 2014. Characterization of polyploid wheat genomic diversity using a high-density 90,000 single nucleotide polymorphism array. Plant Biotechnol. J. 12: 787-796.

William, M., Singh, R. P., Huerta-Espino, J., Ortíz-Islas, S., and Hoisington, D. 2003. Molecular marker mapping of leaf resistance gene $L r 46$ and its association with stripe rust resistance gene $Y r 29$ in wheat. Phytopathology 93 : 153-159.

Windju, S. S., Malla, K., Belova, T., Wilson, R. C., Dieseth, J. A., Alsheikh, M. K., and Lillemo, M. 2017. Mapping and validation of powdery mildew resistance loci from spring wheat cv. Naxos with SNP markers. Mol. Breed. 37:61.

Wu, J. H., Zeng, Q. D., Wang, Q. L., Liu, S. J., Yu, S. Z., Mu, J. M., Huang, S., Sela, H. N., Distelfeld, A., Huang, L. L., Han, D. J., and Kang, Z. S. 2018. SNP-based pool genotyping and haplotype analysis accelerate fine-mapping of the wheat genomic region containing stripe rust resistance gene $\mathrm{Yr} 26$. Theor. Appl. Genet. 131:1481-1496.

Yang, E. N., Li, G. G., Li, L. P., Zhang, Z. Y., Yang, W. Y., Peng, Y. L., Zhu, Y. Q., Yang, Z. J., and Rosewarne, G. M. 2016. Characterization of stripe rust resistance genes in the wheat cultivar Chuanmai45. Int. J. Mol. Sci. 17:601-610.

Zhang, H. Q., Lang, J., Ma, S. Q., and Zhang, B. S. 2008. Genetic analysis and SSR mapping on an new stripe rust resistance gene $\mathrm{YrY}$ 206 in Aegilops tauschii. Chin. J. Biotechnol. 24:1475-1479.

Zhang, X., Han, D., Zeng, Q., Duan, Y., Yuan, F., Shi, J. D., Wang, Q. L., Wu, J. H., Huang, L. L., and Kang, Z. S. 2013. Fine mapping of wheat stripe rust resistance gene $\mathrm{Yr} 26$ based on collinearity of wheat with Brachypodium distachyon and rice. PLoS One 8:e57885.

Zheng, S. G., Li, Y. F., Lu, L., Liu, Z. H., Zhang, C. H., Ao, D. H., Li, L. R., Zhagn, C. Y., Liu, R., Luo, C. P., Wu, Y., and Zhang, L. 2017. Evaluating the contribution of $Y r$ genes to stripe rust resistance breeding through marker-assisted detection in wheat. Euphytica 213:50. 